\title{
Channel aware scheduling for broadcast MIMO systems with orthogonal linear precoding and fairness constraints
}

\author{
G. Primolevo, O. Simeone and U. Spagnolini \\ Dip. di Elettronica e Informazione, Politecnico di Milano \\ P.zza L. da Vinci, 32 I-20133 Milano (Italy).
}

\begin{abstract}
In the downlink of a broadcast fading channel, the base station can capitalize on multiuser diversity through channel aware scheduling. In MIMO systems, the design of the scheduler has to take into account the processing performed at the trasmitter and the receivers.

In this work, we consider channel aware scheduling for orthogonal linear precoding at the base station that guarantees interference free reception for each scheduled users. The problem is set in a novel mathematical framework and a scheduling algorithm is proposed that is shown by simulation to guarantee superior performance as compared to know techniques. Moreover, fairness constraints inspired by the proportional fair criterion are introduced in the scheduling process in order to guarantee the desired long term fairness properties.
\end{abstract}

\section{INTRODUCTION}

In the downlink of a broadcast fading channel, the base station can capitalize on multiuser diversity provided by independent fading realizations across different users. Channel aware scheduling is a technique that allows to achieve this goal by appropriately timing transmission to a subset of one or more users in each available time (code/frequency) slot. Scheduling is performed according to the knowledge of the channel state information available at the scheduler with the general goal of granting transmission to the users that have instantaneous channel near the peak [1].

If base station and users are equipped with a single antenna, it has been shown that transmission to the user with the strongest channel is a strategy that achieves channel capacity [2]. However, if the base station is equipped with an antenna array, more users can be served simultaneously in the same time slot. In particular, if the base station has $n_{T}$ antennas, up to $n_{T}$ users can be allocated in the same time slot with controlled interference. In this case, transmission to a single user is not the optimal solution and the design of the scheduler becomes more complicated depending on the beamforming and power allocation strategy [3] [4].

In MIMO systems (i.e., antenna array at both base station and terminals), the scheduler can leverage on another degree of freedom since each user can be assigned to multiple spatial channels [5]. In fact, if each user has $n_{R}$ receiving antennas $\left(n_{R} \leq n_{T}\right)$, the base station can grant up to $n_{R}$ spatial channels (out of the available $n_{T}$ ) to any user [6]. The design of the scheduler has to take into account the processing performed at the transmitter (e.g., linear precoding and power allocation) and the receivers (e.g., linear equalizer).

Scheduling with linear processing at the base station that simply associates each spatial channel with a transmitting antenna and linear interfaces at the receivers has been considered in [5] (zero forcing equalizer) and [7] (MMSE equalizer). In this paper, we consider channel aware scheduling for

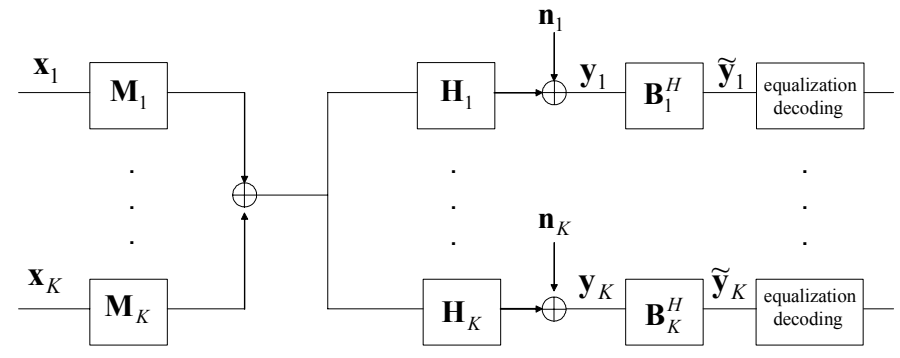

Fig. 1. Block diagram of a broadcast channel with linear interfaces at the transmitter (base station) and receivers (users).

orthogonal linear precoding at the base station and linear zero-forcing equalizers at the receivers (Orthogonal Space Division Multiple Access, OSDMA [8]). Using the OSDMA transmitting/receiving strategy, the spatial channels intended for a given user do not interfere neither with the signal destined to other users nor among themselves.

Channel aware scheduling for OSDMA has been first studied in [8]. We set the problem in a novel mathematical framework and propose a scheduling algorithm that is shown by simulation to guarantee superior performance in terms of sum capacity. Moreover, fairness constraints inspired by the proportional fair criterion [9] [10] are introduced in the scheduling process in order to guarantee the desired long term fairness properties.

\section{SignAL MODEL AND PROBLEM FORMULATION}

The broadcast channel with linear interfaces at the transmitter and receivers is depicted in fig. 1 . Let $\breve{K}$ be the set of $\breve{K}$ available users. The $i$ th user is equipped with an antenna array of $n_{R, i}$ elements and the base station with $n_{T}$ antennas. The subset of $K(t)$ users that are served by the base station within the $t$ th time slot is denoted as $\mathcal{K}(t) \subseteq \breve{\mathcal{K}}$ and its element indexed by $k=1,2, \ldots, K(t)$.

The scheduler allocates $d_{k}(t) \leq n_{R, k}$ spatial channels to the $k$ th user so that all the available $n_{T}$ spatial channels are used:

$$
\sum_{\bar{k}=1}^{K(t)} d_{k}(t)=n_{T} .
$$

The signal intended for the $k$ th user, collected in the $d_{k}(t) \times 1$ vector $\mathbf{x}_{k}(t)$ is linearly precoded by the $n_{T} \times d_{k}(t)$ matrix $\mathbf{M}_{k}(t)$. Following the conventional notation (see, e.g., [6]) and referring to fig. 1, the signal received by the $k$ th user across its $n_{R, k}$ receiving antennas within the $t$ th time slot can 
be written as the $n_{R, k} \times 1$ vector $\mathbf{y}_{k}(t)$

$$
\mathbf{y}_{k}(t)=\mathbf{H}_{k}(t) \mathbf{M}_{k}(t) \mathbf{x}_{k}(t)+\sum_{\substack{i \neq k \\ i \in \mathcal{K}(t)}} \mathbf{H}_{k}(t) \mathbf{M}_{i}(t) \mathbf{x}_{i}(t)+\mathbf{n}_{k}(t)
$$

where $\mathbf{H}_{k}(t)$ is the $n_{R, k} \times n_{T}$ channel matrix of the $k$ th user and $\mathbf{n}_{k}(t)$ is the zero mean additive Gaussian noise with $E\left[\mathbf{n}_{k}(t) \mathbf{n}_{k}(t)^{H}\right]=\sigma_{n}^{2} \mathbf{I}_{n_{R, k}}$.

The received signal $\mathbf{y}_{k}(t)$ lies in a $n_{R, k}$-dimensional linear space. However, only $d_{k}(t) \leq n_{R, k}$ spatial channels are assigned to the $k$ th user. Therefore, the useful part of the received signal spans a $d_{k}(t)$-dimensional subspace that we refer to as receiving subspace. In order to account for this, at the receiver, the $n_{R, k} \times 1$ received signal $\mathbf{y}_{k}(t)$ is pre-filtered by the $d_{k}(t) \times n_{R, k}$ matrix $\mathbf{B}_{k}(t)^{H}$

$$
\begin{aligned}
\tilde{\mathbf{y}}_{k}(t)= & \mathbf{B}_{k}(t)^{H} \mathbf{y}_{k}(t)=\tilde{\mathbf{H}}_{k}(t) \mathbf{M}_{k}(t) \mathbf{x}_{k}(t)+ \\
& +\sum_{\substack{i \neq k \\
i \in \mathcal{K}(t)}} \tilde{\mathbf{H}}_{k}(t) \mathbf{M}_{i}(t) \mathbf{x}_{i}(t)+\tilde{\mathbf{n}}_{k}(t),
\end{aligned}
$$

where we have defined the $d_{k}(t) \times n_{T}$ equivalent channel $\tilde{\mathbf{H}}_{k}(t)=\mathbf{B}_{k}(t)^{H} \mathbf{H}_{k}(t)$ and $\tilde{\mathbf{n}}_{k}(t)=\mathbf{B}_{k}(t)^{H} \mathbf{n}_{k}(t)$. In order to simplify the analysis and without limiting the generality of the approach, we assume

$$
\mathbf{B}_{k}(t)^{H} \mathbf{B}_{k}(t)=\mathbf{I}_{d_{k}(t)}
$$

so that $E\left[\tilde{\mathbf{n}}_{k}(t) \tilde{\mathbf{n}}_{k}(t)^{H}\right]=\sigma_{n}^{2} \mathbf{I}_{d_{k}(t)}$. The range space of $\mathbf{B}_{k}(t)$ corresponds to the receiving subspace for the $k$ th terminal. As a last step, equalization and detection is performed on $\tilde{\mathbf{y}}_{k}(t)$.

In this work, we assume that the channel matrices $\mathbf{H}_{k}(t)$ are known to the transmitter and receivers, e.g., by transmission of pilot symbols and feedback of the channel state information from the receivers to the base station [11]. An analysis of the effect of imperfect channel state information and feedback delays is proposed in [12].

In order to simplify the notation, in the following the temporal dependence on $t$ is omitted.

\section{A. Problem formulation}

In principle, we would like to find the subset of users $\mathcal{K}$ and the set of precoding matrices $\mathcal{M}=\left\{\mathbf{M}_{i}\right\}_{i=1}^{\breve{K}}$ and pre-filtering matrices $\mathcal{B}=\left\{\mathbf{B}_{i}\right\}_{i=1}^{\breve{K}}$ so that the sum capacity is maximized under a total power constraint (recall also constraints (1) and (4)):

$$
\begin{gathered}
\{\mathcal{B}, \mathcal{M}\}=\underset{\mathcal{B}, \mathcal{M}}{\operatorname{argmax}} \sum_{i=1}^{\breve{K}} C_{i}(\mathcal{B}, \mathcal{M}) \\
\text { s.t. } \sum_{i=1}^{\breve{K}} \operatorname{tr}\left(\mathbf{M}_{i} \mathbf{M}_{i}^{H}\right) \leq \bar{P},
\end{gathered}
$$

where $C_{i}(\mathcal{B}, \mathcal{M})$ is the link capacity for the $i$ th user [6]

$$
C_{i}(\mathcal{B}, \mathcal{M})=\log _{2}\left|\mathbf{I}_{d_{i}}+\mathbf{R}_{i}^{-1}\left(\tilde{\mathbf{H}}_{i} \mathbf{M}_{i} \mathbf{M}_{i}^{H} \tilde{\mathbf{H}}_{i}^{H}\right)\right|
$$

with

$$
\mathbf{R}_{i}=\sigma_{n}^{2} \mathbf{I}_{d_{i}}+\sum_{\substack{k \neq i \\ k \in \mathcal{K}}} \mathbf{H}_{i} \mathbf{M}_{k} \mathbf{M}_{k}^{H} \mathbf{H}_{i}^{H}
$$

In (6)-(7) the assumption of Gaussian codebooks with $E\left[\mathbf{x}_{i} \mathbf{x}_{i}^{H}\right]=\mathbf{I}_{d_{i}}$ is implied. Moreover, as a result of the optimization problem (5) the $i$ th user belongs to the set of active users $\mathcal{K}$ if $d_{i}>0$ or equivalently $\left(\mathbf{M}_{i}, \mathbf{B}_{i}\right)$ are not empty matrices.

Solution of the optimization problem (5) is not known, even for the case of given sets $\mathcal{K}$ and $\mathcal{B}$. In [8], an algorithm is proposed for obtaining an approximate solution based on the additional constraint of zero inter-user interference and the separate computation of precoding and scheduling as explained in Sec. III. The treatment is aimed at setting the results of [8] in the discussed mathematical framework and review the main concepts. A novel approximate solution of (5) is then proposed in Sec. IV. The algorithm is still based on the inclusion of the zero inter-user interference constraint but, differently from [8], it performs jointly precoding and scheduling.

\section{REVIEW OF MIMO-OSDMA WITH LSV SCHEDULING}

According to the approximate solution of (5) proposed in [8], at first the scheduling step is performed. This amounts to select the subset $\mathcal{K}$ and the corresponding $K$ matrices $\mathbf{B}_{k}$. Recall that the choice of $\mathbf{B}_{k}$ implies the allocation of $d_{k}$ spatial channel to the $k$ th user and the corresponding receiving subspace. Then, the design of the precoding matrices $\mathbf{M}_{k}$ is carried out with the additional constraint of granting inter-user interference free transmission (MIMO-OSDMA).

\section{A. Largest Singular Value (LSV) channel aware scheduling}

In [8], selection of the subset $\mathcal{K}$ and of the corresponding $K$ matrices $\left\{\mathbf{B}_{k}\right\}_{k=1}^{K}$ is performed so as to set as active the spatial channels corresponding to the largest singular values of matrices $\left\{\mathbf{H}_{i}\right\}_{i=1}^{\breve{K}}$. To elaborate, let $\lambda_{i, j}, j=1, \ldots, r_{i}=$ $\operatorname{rank}\left(\mathbf{H}_{i}\right)$ be the non-zero singular values of channel matrix $\mathbf{H}_{i}$ gathered in the diagonal matrix $\boldsymbol{\Lambda}_{i}$ and $\left(\mathbf{u}_{i, j}, \mathbf{v}_{i, j}\right)$ the corresponding left and right singular vectors collected by columns in matrices $\mathbf{U}_{i}$ and $\mathbf{V}_{i}$ respectively: $\mathbf{H}_{i}=\mathbf{U}_{i} \boldsymbol{\Lambda}_{i} \mathbf{V}_{i}^{H}$. The LSV algorithm selects the $n_{T}$ largest singular values of the set $\left\{\lambda_{i, j} \mid i=1, \ldots, \breve{K}, j=1, \ldots, r_{i}\right\}$ and builds matrices $\mathbf{B}_{k}$ with the corresponding left singular vectors $\mathbf{v}_{i, j}$.

This algorithm can equivalently be stated as the solution of the following optimization problem: find the set $\mathcal{B}=\left\{\mathbf{B}_{i}\right\}_{i=1}^{\breve{K}}$ so that (recall also constraints (1) and (4)):

$$
\mathcal{B}=\underset{\mathcal{B}}{\operatorname{argmax}} \sum_{i=1}^{\breve{K}}\left\|\mathbf{B}_{i}^{H} \mathbf{H}_{i}\right\|^{2}
$$

Notice that an user belongs to $\mathcal{K}$ if the corresponding number of assigned channel $d_{i}$ is not zero, or equivalently $\mathbf{B}_{i}$ is not empty.

\section{B. MIMO Orthogonal Space Division Multiple Access (OS- $D M A)$}

Given the output of the scheduling algorithm (i.e., the set $\mathcal{K}$ and matrices $\left.\left\{\mathbf{B}_{k}\right\}_{k=1}^{K}\right)$, the precoding matrices $\mathbf{M}_{k}$ are derived by maximizing the sum capacity (5) with an additional zero interference constraint among the users. Notice that the 
zero-interference assumption is capacity achieving in the high signal-to-noise ratio regime where the interference plays a major role in defining the system performance. In particular, the following optimization problem is solved:

$$
\begin{gathered}
\mathcal{M}=\underset{\mathcal{M}}{\operatorname{argmax}} \sum_{i=1}^{K} C_{i}(\mathcal{B}, \mathcal{M}) \\
\text { s.t. } \sum_{k=1}^{K} \operatorname{tr}\left(\mathbf{M}_{k} \mathbf{M}_{k}^{H}\right) \leq P, \\
\tilde{\mathbf{H}}_{i} \mathbf{M}_{j}=\mathbf{0} \text { if } i \neq j,
\end{gathered}
$$

Constraint (9c) ensures zero-interference among the $K$ active users. Therefore, the capacity for the $i$ th user can be written as (6) with $\mathbf{R}_{i}=\sigma_{n}^{2} \mathbf{I}_{d_{i}}$.

The resulting precoding matrix $\mathbf{M}_{k}$ has the following form [8]

$$
\mathbf{M}_{k}=\Theta_{k} \mathbf{P}_{k}
$$

where $\Theta_{k}$ is a $n_{T} \times d_{k}$ matrix is selected as

$$
\Theta_{k}=\overline{\mathbf{V}}_{k}^{0} \tilde{\mathbf{V}}_{k}
$$

where $\overline{\mathbf{V}}_{k}^{0}$ is a $n_{T} \times d_{k}$ basis of the $d_{k}$-dimensional null space of the $n_{T} \times\left(n_{T}-d_{k}\right)$ matrix $\overline{\mathbf{H}}_{k}^{H}$ with the singular value decomposition

$$
\begin{aligned}
\overline{\mathbf{H}}_{k} & =\left[\tilde{\mathbf{H}}_{1}^{H} \cdots \tilde{\mathbf{H}}_{k-1}^{H} \tilde{\mathbf{H}}_{k}^{H} \cdots \tilde{\mathbf{H}}_{K}^{H}\right]^{H}= \\
& =\left[\overline{\mathbf{U}}_{i} \overline{\mathbf{U}}_{i}^{0}\right]\left[\begin{array}{cc}
\overline{\mathbf{\Lambda}}_{i} & \mathbf{0} \\
\mathbf{0} & \mathbf{0}
\end{array}\right]\left[\overline{\mathbf{V}}_{i} \overline{\mathbf{V}}_{i}^{0}\right]^{H},
\end{aligned}
$$

this guarantees the zero inter-user interference constraint (9c). Matrix $\tilde{\mathbf{V}}_{k}$ is the $d_{k} \times d_{k}$ range space of $\tilde{\mathbf{H}}_{k}^{H}$, thus ensuring zero interference among all the spatial channels. Finally, $\mathbf{P}_{k}$ is a $d_{k} \times d_{k}$ diagonal matrix that defines power allocation over the spatial channels selected according to the multi-user waterfilling criterion.

\section{MIMO-OSDMA WITH SVS SCHEDULING}

The approximate solution of the problem (9) proposed by [8] suffers from degraded performance (as it will be shown in Sec. VI) mainly because the precoding matrices $\mathbf{M}_{k}$ and the prefiltering matrices $\mathbf{B}_{k}$ (and the associated set $\mathcal{K}$ ) are optimized separately. Here we propose a joint optimization that approximates problem (9) as follows. i) The zero interference constraint $(9 \mathrm{c})$ is imposed, thus obtaining a MIMOOSDMA system as in [8]. As explained in Sec. III-B, the resulting precoding matrices have the form (10)-(11). ii) The objective function $C_{i}(\mathcal{B}, \mathcal{M})$ is approximated by its first term of the Taylor expansion: $C_{i}(\mathcal{B}, \mathcal{M}) \approx 1 / \sigma_{n}^{2}\left\|\mathbf{B}_{i}^{H} \mathbf{H}_{i} \mathbf{M}_{i}\right\|^{2}$. The latter approximation is expected to hold at sufficiently low signal-to-noise ratios. The resulting optimization problem reads (recall also constraints (1) and (4) and define $\overline{\mathcal{V}}^{0}=$ $\left.\left\{\overline{\mathbf{V}}_{i}^{0}\right\}_{i=1}^{K}\right)$ :

$$
\begin{gathered}
\left\{\mathcal{B}, \overline{\mathcal{V}}^{0}\right\}=\underset{\mathcal{B}, \overline{\mathcal{V}}^{0}}{\operatorname{argmax}} \sum_{i=1}^{\breve{K}} N_{i}\left(\mathcal{B}, \overline{\mathcal{V}}^{0}\right), \\
\text { s.t. } \quad \tilde{\mathbf{H}}_{i} \overline{\mathbf{V}}_{j}^{0}=\mathbf{0}, i \neq j,
\end{gathered}
$$

where we defined $N_{i}\left(\mathcal{B}, \overline{\mathcal{V}}^{0}\right)=\left\|\mathbf{B}_{i}^{H} \mathbf{H}_{i} \overline{\mathbf{V}}_{i}^{0}\right\|^{2}$. The objective function (13a) is amenable to an efficient numerical optimization and will be shown in Sec. VI to yield relevant advantages as compared to the separate optimization proposed in [8]. Notice that in order to simplify the solution of the problem, the remaining term of the precoding matrices (10), $\tilde{\mathbf{V}}_{k}$ and $\mathbf{P}_{k}$, are assumed to be computed according to Sec. III-B, thus guaranteeing zero interference among different streams and the enforcement of the total power constraint.

\section{A. Successive Vector Selection (SVS) channel aware schedul- ing}

Problem (13) can be efficiently solved by a greedy approach as detailed in the following. The idea is to select at each step the spatial channel that yields the largest increase of the objective function (13a). Let us denote with the superscript $(n)$ the quantities of interest as computed at the $n$th iteration. At each iteration a spatial channel (out of the $n_{T}$ available) is allocated to a specific user so that a total number of $n_{T}$ iterations are needed. We are interested in updating the receiving subspaces $\mathbf{B}_{i}$ (initialized as $\mathbf{B}_{i}^{(0)}$ equal to an empty matrix) and the transmitting subspaces $\overline{\mathbf{V}}_{i}^{0}$, or equivalently its orthogonal complement $\overline{\mathbf{V}}_{i}$ (see (12), initialization: $\overline{\mathbf{V}}_{i}^{0(0)}=$ $\left.\mathbf{I}_{n_{T}}\right)$. Let $\mathbf{u}_{j}$ be a possible candidate vector to be included in the receiving subspace $\mathbf{B}_{j}^{(n)}$ of user $j$ at the $n$th iteration $(j=1, \ldots, \breve{K})$. As a result of the choice of $\mathbf{u}_{j}$ at the $n$th iteration, the objective function (13a) modifies as (dropping the functional dependence on $\mathcal{B}, \overline{\mathcal{V}}^{0}$ for simplicity of notation)

$$
\sum_{i=1}^{\breve{K}} N_{i}^{(n)}\left(\mathbf{u}_{j}\right)=\sum_{i=1}^{\breve{K}} N_{i}^{(n-1)}+\sum_{i=1}^{\breve{K}} \Delta N_{i}^{(n)}\left(\mathbf{u}_{j}\right) .
$$

Among all the possible vectors $\mathbf{u}_{j}$ for all users $j=1, \ldots, \breve{K}$, the vector $\mathbf{u}_{j}$ is selected so as to maximize the increase of the objective function $\sum_{i=1}^{\breve{K}} \Delta N_{i}^{(n)}\left(\mathbf{u}_{j}\right)$. In the following, the computation of $\Delta N_{i}^{(n)}\left(\mathbf{u}_{j}\right)$ is carried out.

To elaborate, we need to define for each user a basis $\mathbf{U}_{j}^{(n)}$ that spans the range space of the channel matrix $\mathbf{H}_{j}$ that at the $n$th iteration has not be assigned to any receiving subspace. Formally, it is: $\operatorname{span}\left\{\mathbf{U}_{j}^{(n)}\right\}=\operatorname{span}\left\{\mathbf{U}_{j}\right\} \cap \operatorname{null}\left\{\mathbf{B}_{j}^{(n)}\right\}$. Therefore, the corresponding initialization is $\mathbf{U}_{j}^{(0)}=\mathbf{U}_{j}$. At the $n$th iteration we have $\sum_{i=1}^{\breve{K}} \hat{d}_{i}^{(n)}=n$ and the possible candidate vectors to be included in the receiving subspace of the $j$ th user are linear combinations of the columns of $\mathbf{U}_{j}^{(n)}$ :

$$
\mathbf{u}_{j}=\mathbf{U}_{j}^{(n)} \mathbf{a}_{j}, \text { with }\left\|\mathbf{a}_{j}\right\|^{2}=1 .
$$

With the selection of (15), the corresponding receiving subspace is updated as $\mathbf{B}_{j}^{(n)}=\left[\mathbf{B}_{j}^{(n-1)} \mathbf{u}_{j}\right]$ while its transmit subspace remains unchanged, $\overline{\mathbf{V}}_{j}^{0(n)}=\overline{\mathbf{V}}_{j}^{0(n-1)}$, since no new constraint (9c) is imposed upon it. It follows that

$$
\Delta N_{j}^{(n)}\left(\mathbf{u}_{j}\right)=\left\|\mathbf{u}_{j}^{H} \mathbf{H}_{j} \overline{\mathbf{V}}_{j}^{0(n)}\right\|^{2}
$$

Then, let $\mathbf{v}_{j}=\mathbf{H}_{j}^{H} \mathbf{u}_{j}$ be the vector corresponding to $\mathbf{u}_{j}$ on the transmitter side. The choice of $\mathbf{u}_{j}$ for user $j$ results in an 
additional zero-interference constraint for any user $i \neq j$ (see (13)), that leads to

$$
\overline{\mathbf{V}}_{i}^{(n)}=\left[\overline{\mathbf{V}}_{i}^{(n-1)} \mathbf{w}_{i}\right],
$$

where $\mathbf{w}_{i}$ is the projection of $\mathbf{v}_{j}$ over $\overline{\mathbf{V}}_{i}^{0(n-1)}$, scaled to unit length

$$
\mathbf{w}_{i}=\left(\overline{\mathbf{V}}_{i}^{0(n-1)} \overline{\mathbf{V}}_{i}^{0(n-1) H} \mathbf{v}_{j}\right) /\left\|\overline{\mathbf{V}}_{i}^{0(n-1)} \overline{\mathbf{V}}_{i}^{0(n-1) H} \mathbf{v}_{j}\right\| .
$$

$\overline{\mathbf{V}}_{i}^{0(n)}$ is updated as well, so that $\operatorname{span}\left(\overline{\mathbf{V}}_{i}^{0(n)}\right)=\operatorname{null}\left(\overline{\mathbf{V}}_{i}^{(n)}\right)$. This step can be performed, e.g., by updating the QR decomposition of (17) [13]. On the other hand, nothing changes at the receiver side of the $i$ th user, $\mathbf{B}_{i}^{(n)}=\mathbf{B}_{i}^{(n-1)}$. It is easy to show that

$$
\Delta N_{i}^{(n)}\left(\mathbf{u}_{j}\right)=-\left\|\mathbf{B}_{i}^{(n) H} \mathbf{H}_{i} \mathbf{w}_{i}\right\|^{2} \quad i \neq j .
$$

To sum up, from (16) and (19) the increase of objective function (13) due to the choice of vector $\mathbf{u}_{j}$ at the $n$th iteration is

$$
\sum_{i=1}^{\breve{K}} \Delta N_{i}^{(n)}\left(\mathbf{u}_{j}\right)=\left\|\mathbf{u}_{j}^{H} \mathbf{H}_{j} \overline{\mathbf{V}}_{j}^{0(n)}\right\|^{2}-\sum_{i \neq j}\left\|\mathbf{B}_{i}^{(n) H} \mathbf{H}_{i} \mathbf{w}_{i}\right\|^{2} .
$$

The first term in (20) accounts for the increased useful power received by user $j$ on the newly assigned spatial channel, whereas the other terms represent the power loss suffered from the other users from not being allowed to transmit over $\mathbf{w}_{i}$ anymore. Recalling (18) and (15), function (20) can be easily recognized to be a sum of Rayleigh quotients in terms of vector $\mathbf{a}_{j}$. While the maximization of a single Rayleigh quotient is analytically feasible since it corresponds to the solution of a generalized eigenvalue problem, maximizing a sum of Rayleigh quotients is much more difficult and costly. Here, we resort to a sub-optimal approach, by restricting $\mathbf{a}_{j}$ to be a column of an identity matrix, which translates to restricting our search of the optimal $\mathbf{u}_{j}$ to the columns of $\mathbf{U}_{j}^{(n)}$. This approach has been proved by simulation to yield performance very close to the optimum solution.

\section{SVS ALGORITHM WITH PROPORTIONAL FAIRNESS CONSTRAINTS}

The algorithms discussed so far aim to maximize the system throughput. If the users are unbalanced, with some of them experiencing strongly attenuated channels, it is expected that the algorithms will result in an unfair sharing of system resources that might preclude communication to some users (see also Sec. VI). Similarly to the proportional fair criterion [10], a scheduling procedure that achieves over a long term an appropriate balance between sum capacity and fairness among users can be defined by modifying (13) as follows (here we explicit for convenience the time dependence):

$$
\begin{gathered}
\left\{\mathcal{B}(t), \overline{\mathcal{V}}^{0}(t)\right\}=\underset{\mathcal{B}, \overline{\mathcal{V}}^{0}}{\operatorname{argmax}} \sum_{i=1}^{\breve{K}} \log \left(E\left[N_{i}\left(\mathcal{B}, \overline{\mathcal{V}}^{0}\right)\right]\right), \\
\text { s.t. } \quad \tilde{\mathbf{H}}_{i}(t) \overline{\mathbf{V}}_{j}^{0}(t)=\mathbf{0}, i \neq j .
\end{gathered}
$$

where $E[\cdot]$ refers to the long term average over time. Following the analysis of [9] and the considerations in Sec. IV , it can be shown that a procedure that (approximately) converges to the solution of (21) can be obtained by implementing the SVS algorithm on normalized channel matrices

$$
\mathbf{H}_{i}^{N}(t)=\frac{\mathbf{H}_{i}(t)}{\alpha_{i}(t)}
$$

with

$$
\alpha_{i}(t)=\left(1-\frac{1}{t_{c}}\right) \hat{\alpha}_{i}(t)+\frac{1}{t_{c}} N_{i}\left(\mathcal{B}(t-1), \overline{\mathcal{V}}^{0}(t-1)\right),
$$

where parameter $t_{c}$ rules the memory of the algorithm. Parameter $\alpha_{i}(t)$ measures the channel power that each user has been allowed to use within a window of $t_{c}$ time slots. The rationale of the algorithm is that if a given user has been ignored by the scheduling procedure in the considered time window, the matrix scaling (22) will force the SVS algorithm to allocate resources to it.

The criterion (21) is a fairness constraints on the channel norms: its implication on the channel rates is not obvious and will be investigated in the next Section by numerical simulations.

\section{NUMERICAL SIMULATIONS}

The performance of the proposed SVS algorithm is compared with the LSV algorithm [8] by Monte Carlo simulations. We consider $\breve{K}=4$ users, where each user has the same number of receiving antennas $n_{R_{i}}=2$ while the base station is equipped with $n_{T}=4$ antennas. Where not stated otherwise, the channels are assumed to be subject to identically distributed Raleigh fading, $\operatorname{vec}\left(\mathbf{H}_{i}\right) \sim \mathcal{C N}\left(0, \mathbf{I}_{n_{T} n_{R_{i}}}\right)$. As reference performance, a random user selection algorithm is considered that chooses randomly a set $\mathcal{K}$ of users such that (1) is satisfied. On this subset, orthogonal precoding is applied as detailed in Sec. III-B. Moreover, the performance of a $n_{T} \times n_{T}$ single user MIMO link is evaluated in order to set a reference level for the sum capacity of the multiuser system.

The ergodic sum capacity is plotted versus the signal to noise ratio $P / \sigma_{n}^{2}$ in fig. 2 . The proposed SVS algorithm yields a gain of about $4 d B$ as compared to the LSV algorithm, whose performance are, in this case, similar to random users selection. As explained in Sec. IV, the advantage of SVS is due to the joint computation of the transmitting and receiving subspaces.

Fig. 3 shows sum capacity versus outage probability for $P / \sigma_{n}^{2}=10 d B$. It can be seen that the slope of the outage probability for SVS is comparable to that of a single user channel, proving the ability of the SVS algorithm to appropriately exploit the diversity of the broadcast channel.

Let us now consider unbalanced users in order to validate the performance of the SVS algorithm with fairness constraints. To be specific, the channels are assumed to be selected so that $\operatorname{vec}\left(\mathbf{H}_{i}\right) \sim \mathcal{C N}\left(0, \beta_{i} \mathbf{I}_{n_{T} n_{R_{i}}}\right)$, where $\beta_{1}=0 d B$, $\beta_{2}=-5 d B, \beta_{3}=-10 d B$ and $\beta_{4}=-20 d B$. The performance of the SVS algorithm is evaluated with and without fairness constraints $\left(t_{c}=20\right)$. The results are summarized in fig. 4 in terms of ergodic sum capacity and individual 


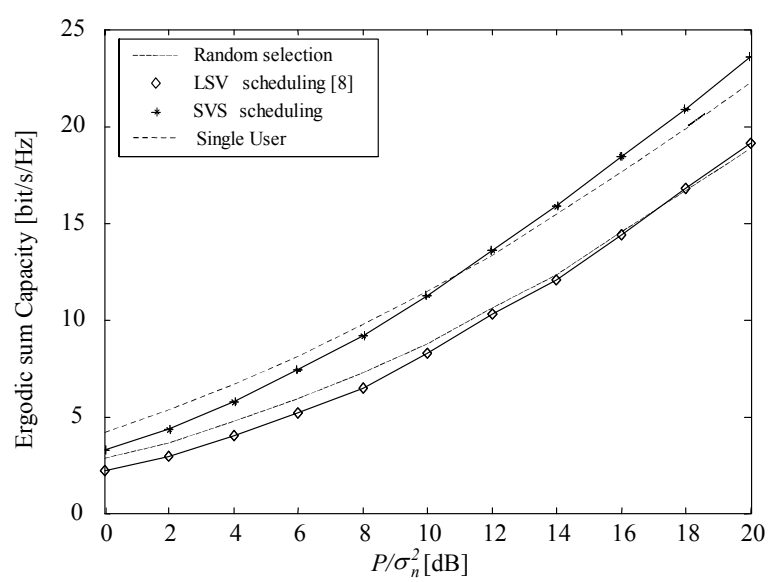

Fig. 2. Ergodic sum capacity versus $P / \sigma_{n}^{2}\left(K=4, n_{T}=4, n_{R}=2\right)$.

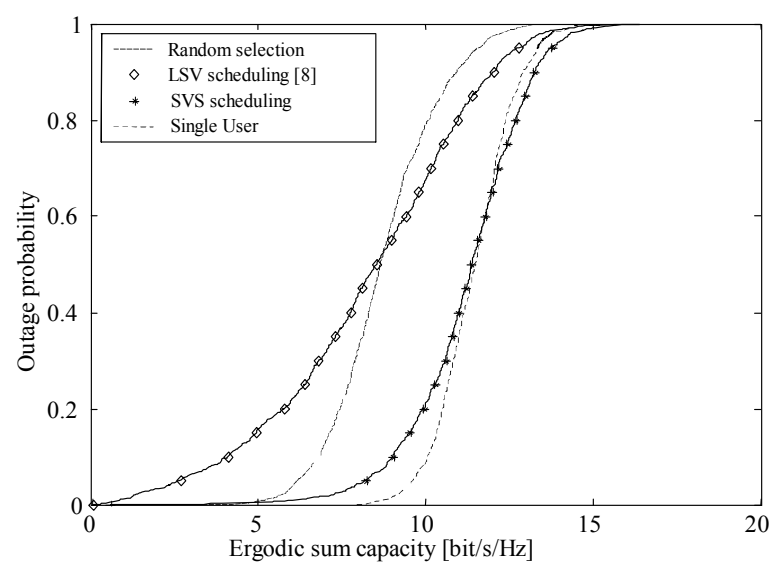

Fig. 3. Sum capacity versus outage probability $\left(K=4, n_{T}=4, n_{R}=2\right.$, $\left.P / \sigma_{n}^{2}=10 d B\right)$.

ergodic capacity versus time $t$. The total throughput loss increases as the fairness constraints are imposed by the scaling algorithm discussed in Sec. V and converges to approximately $2 b i t / s / H z$. This decrease of the sum capacity translates in a more fair sharing of resources as proved by the individual channel capacities. Notice that the channel capacities for $t=0$ correspond to the performance of the SVS algorithm with no fairness constraints. Even though the fairness constraint (21) was imposed on the channel norms, rather than on the individual rates as in [10], the simulation results indicate that the proportional fair criterion is very closely followed by the channel capacities as well. In fact, the user capacities approximately converge to the dashed lines in fig. 4 that denote the individual capacities as obtained by sharing the long term sum capacity according to the proportional fair criterion (i.e., in proportion to the single user capacities $E\left[\log _{2} \mid \mathbf{I}_{d_{i}}+P / K\right.$. $\left.\left.\mathbf{H}_{i} \mathbf{H}_{i}^{H} \mid\right][10]\right)$.

\section{CONCLUSION}

The problem of channel aware scheduling for broadcast MIMO channels with orthogonal linear precoding and linear interfaces at the receivers has been investigated. An algorithm

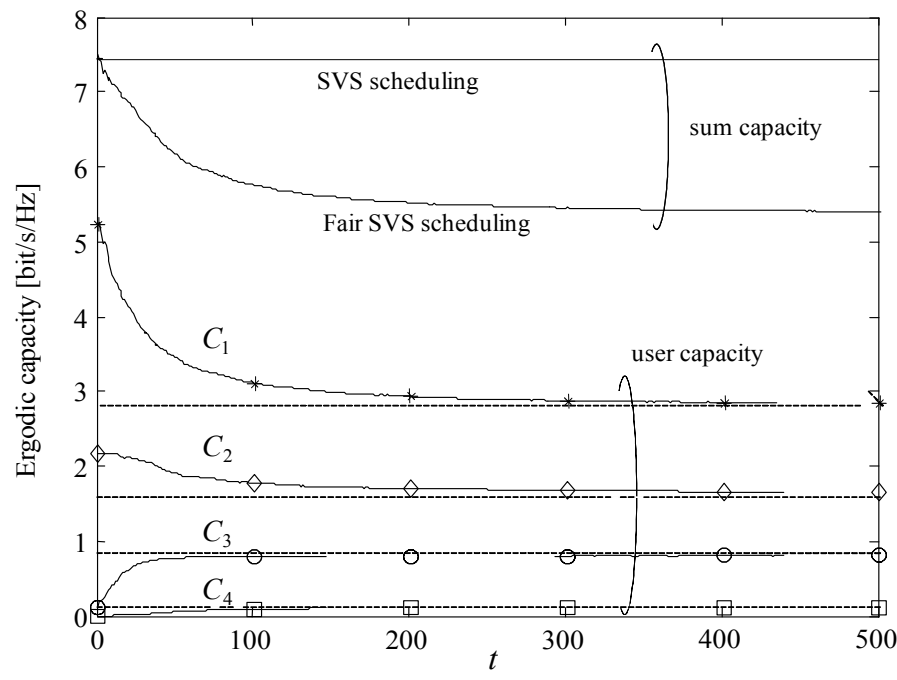

Fig. 4. Ergodic sum capacity and individual capacities versus time $t$ for the SVS algorithm with and without fairness constraints (unbalanced users, $\left.K=4, n_{T}=4, n_{R}=2, P / \sigma_{n}^{2}=10 d B\right)$.

based on the joint optimization of precoding and scheduling has been proposed and its performance proved by simulation to be superior to know techniques in terms of sum capacity. Moreover, the introduction of proportional fairness constraints has been discussed and a modification of the algorithm that yields desirable long term fairness properties has been proposed and validated through simulation.

\section{REFERENCES}

[1] R. Knopp and P. Humblet, "Information capacity and power control in single cell multiuser communications," Proc. IEEE Int. Computer Conf., pp. 331-335, June 1995.

[2] D. N. C. Tse, "Optimal power allocation over parallel Gaussian channels," Proc. IEEE ISIT, p. 27, 1997.

[3] H. Viswanathan and K. Kumaran, "Rate scheduling in multiple antenna downlink wireless systems," Proc. Allerton Conference, 2001.

[4] I. Koutsopoulos, T. Ren and L. Tassiulas, "The impact of space division multiplexing on resource allocation: a unified view," Proc. IEEE Infocom, pp. 533-543, 2003.

[5] R. W. Heath Jr., M. Airy, A. J. Paulraj, "Multiuser diversity for MIMO wireless systems with linear receivers," Proc. Asilomar Conf. Signals, Systems and Computers, pp. 1194-1199, Nov. 2001.

[6] A. Goldsmith, S. A. Jafar, N. Jindal and S. Vishwanath, "Capacity limits of MIMO channels," IEEE J. Select. Areas Commun., vol. 21, no. 5, pp. 684-702, June 2003.

[7] O. Shin and K. Bok, "Antenna-assisted round robin scheduling for MIMO cellular systems," IEEE Comm. Letters, vol. 7, no. 3, pp. 109111, March 2003.

[8] Q. H. Spencer, A. Lee Swindlehurst and M. Haardt, "Zero-forcing methods for downlink spatial multiplexing in multiuser MIMO channels", IEEE Trans. Signal Processing, vol. 52 , no. 2 , pp. 461-471, Feb. 2004.

[9] H. J. Kushner and P. Whiting, "Convergence of proportional-fair sharing algorithms under general conditions", IEEE Trans. Wireless Commun., vol. 3, no. 4, pp. 1250-1259, July 2004.

[10] P. Viswanath, D. N. C. Tse, R. Laroia, "Opportunistic beamforming using dumb antennas," IEEE Trans. Inform. Theory, vol. 48, no. 6, pp. 1277-1294, June 2002.

[11] T. Marzetta and B. Hochwald, "Fast transfer of channel state information in wireless systems," submitted to IEEE Trans. Signal Processing [also available on http://mars.bell-labs.com/].

[12] G. Primolevo, O. Simeone and U. Spagnolini, "Effects of imperfect channel state information on the capacity of broadcast OSDMA-MIMO systems", Proc. IEEE SPAWC, 2004.

[13] G. H. Golub and C. F. van Loan, Matrix Computations, Johns Hopkins, 1996, 3rd edition. 